\title{
Detection and estimating the blood accumulation volume of brain hemorrhage in a human anatomical skull using a RF single coil
}

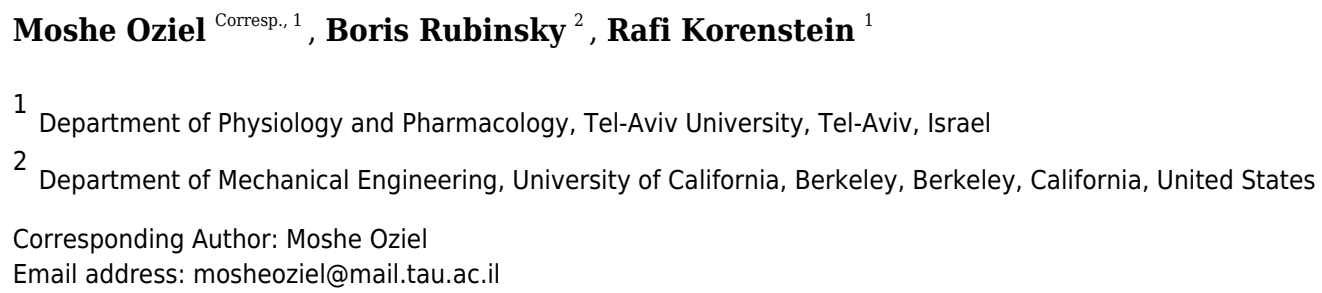

Objective. An experimental study for testing a simple robust algorithm on data derived from an electromagnetic radiation device that can detect small changes in the tissue/fluid ratio in a realistic head configuration. Methods. Changes in the scattering parameters (S21) of an inductive coil resulting from injections of chicken blood in the $0-18 \mathrm{ml}$ range into calf brain tissue in a human anatomical skull were measured over a $100-1000 \mathrm{MHz}$ frequency range. Results. An algorithm that combines amplitude and phase results was found to detect changes in the tissue/fluid ratio with $90 \%$ accuracy. An algorithm that estimated the injected blood volume was found to have a 1-4 $\mathrm{ml}$ average error. This demonstrates the possibility of the inductive coil-based device to possess a practical ability to detect a change in the tissue/fluid ratio in the head. Significance. This study is an important step towards the goal of building an inexpensive and safe device that can detect an early brain hemorrhagic stroke. 
1 Detection and estimating the blood accumulation 2 volume of brain hemorrhage in a human anatomical

\section{skull using a RF single coil}

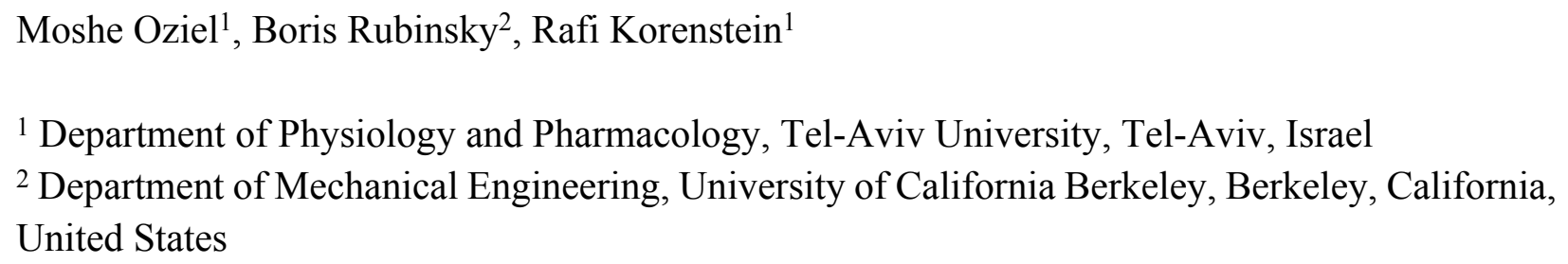

\section{Abstract}

Objective. An experimental study for testing a simple robust algorithm on data derived from an electromagnetic radiation device that can detect small changes in the tissue/fluid ratio in a realistic head configuration.

Methods. Changes in the scattering parameters (S21) of an inductive coil resulting from injections of chicken blood in the $0-18 \mathrm{ml}$ range into calf brain tissue in a human anatomical skull were measured over a $100-1000 \mathrm{MHz}$ frequency range.

Results. An algorithm that combines amplitude and phase results was found to detect changes in the tissue/fluid ratio with $90 \%$ accuracy. An algorithm that estimated the injected blood volume was found to have a 1-4 $\mathrm{ml}$ average error. This demonstrates the possibility of the inductive coilbased device to possess a practical ability to detect a change in the tissue/fluid ratio in the head.

Significance. This study is an important step towards the goal of building an inexpensive and safe device that can detect an early brain hemorrhagic stroke.

\section{Introduction}

The chance of having a hemorrhagic stroke is between $0.01 \%$ and $0.03 \%$ of the worldwide population (Qureshi et al., 2009). The risk of dying a year after a stroke is about 50\% (An et al., 2017). Studies have shown that the volume of bleeding in the brain increases within the first 3 hours of a stroke for $73 \%$ of patients (Davis et al., 2006; Brott et al., 2009) where an increase in bleeding volume is closely linked to lower chances of recovery from the stroke (Davis et al., 2006; Dowlatshahi, 2011). Thus, precise diagnosis and treatment swiftness seem to be important factors in patient survival (Brain Aneurysm Foundation, 2019). 
When a symptomatic patient is brought to a hospital, the hemorrhage can be detected by Magnetic Resonance Imaging (MRI), Computed Tomography (CT), or Doppler Ultrasound (Huisman, 2005). These imaging technologies are accurate and supply the relevant information needed for diagnostics. However, the price of these devices is high and require skilled operators. In the developing world, most of the population has no access to these technologies (González et al., 2010). While CT is commonly used for detecting a hemorrhagic stroke, the possibility to detect temporal hemorrhage is limited to the number of repeat CT scans allowed, in particular for children who are most susceptible to the X-ray radiation employed by the CT (Little, 2008).

A measure for the occurrence of progressive internal bleeding is to detect if the volume ratio of tissue/fluid in an organ changes over time. We have developed several simple, non-contact, and inexpensive radiofrequency (RF) and microwave (MW) frequency based diagnostic technologies for detecting internal bleeding in the body, with a goal towards their use for the economically disadvantaged populations. One of these technologies, referred to as volumetric integral phaseshift spectroscopy (VIPS), monitors the change in the amplitude and phase between two coils across the head in the frequency range of RF (González \& Rubinsky, 2006). In a clinical study with 248 subjects, VIPS has shown that it can detect a severe stroke in seconds with a $92 \%$ accuracy (Kellner et al., 2018) and has been chosen as one of the top ten medical innovations for 2019 (Cleveland Clinic, 2019). VIPS has received FDA and CE approval and is used clinically to detect brain hemorrhages (González et al.,2014), cerebrovascular autoregulation monitoring (Oziel et al., 2016), or to detect fluid shifts in the brain during dialysis (Rao, 2018). There are many other uses of RF/MW measurements for diagnostics in medicine. For example, for monitoring breathing (Richer \& Adler, 2005; Teichmann et al., 2013; Teichmann et al., 2014), magnetic imaging tomography (MIT) (Mobashsher \& Abbosh, 2014) or thermal therapy monitoring (Haynes et al.,2014). The technologies that we have developed emerge from the fact that the intracranial volume is constant, and changes in the ratio of blood/brain tissue structure alter the bulk electromagnetic properties of the entire head (Griffiths et al., 1999; Qureshi et al.2018).

The VIPS technology employs a transmitter coil and one or more receiver coils. While precise, this makes the technology sensitive to motion artifacts, given the major contribution to the signal strength is usually the direct path between the transmitter and receiver. One possible solution is using a single coil, thereby removing the location dependence between two coils. Due to the spatial symmetry of the induction coil (surrounds the head), there is no single path with an extraordinary contribution to the received signal. Therefore, the response to the relative change in the position of the single coil will be less significant than the response to the change in the relative position in a two-coil system. Our previous studies of monitoring the brain by a single coil began with mathematical modeling. We used numerical simulations to analyze the performances of a spiral antenna (Oziel et al., 2017) and a single inductive coil surrounding the head (Oziel et al., 2018). These feasibility studies evaluated the sensitivity of the radar-based measurements to small changes in the blood/tissue volume ratio in the case of a brain hemorrhage. The simulations predicted that these devices can detect changes as small as $2 \mathrm{ml}$ in the blood volume. We also found that a device based on a single-coil is superior to the spiral antenna when the bleeding 
79 location is unknown. In a further evaluation of the technology, we concluded that the single 80 inductive coil is easier and less expensive to manufacture and use than the spiral antenna 81 configuration (Oziel et al., 2018). Next, we performed an experiment on a cylindrically shaped 82 alcohol-based phantom gel that simulates brain tissue, into which we injected a physiological fluid 83 that simulates blood (Oziel et al., 2020a). We also developed a new statistical method to analyze 84 the experiments with this simple configuration. The analysis of these experiments shows that a 85 single coil operating at 100-1000 MHz can detect changes in the tissue/fluid ratio as small as $2 \mathrm{ml}$. 86 We learned that there are only a small number of frequencies that are strongly affected by changes in the tissue/fluid ratio. These frequencies are affected mainly by boundary conditions as well as by the location of the accumulated liquid. We have also shown that for those frequencies, there is a correlation between the changes in amplitude and phase in response to the injection of physiological saline (Oziel et al., 2020a).

Recently, we developed a very simple and robust algorithm for the single inductive coil configuration (Oziel et al., 2020b). The algorithm can detect changes in the liquid/gel ratio in the cylindrical configuration of the head (Oziel et al., 2020a) with very high accuracy. This algorithm was also able to roughly estimate the injection volume.

In the present study, we validate the single-coil based technology of detection internal head bleeding conceived first in previous study (Oziel et al., 2017), on a phantom as close as possible to a real human head. This is an experimental study on a human anatomical shape skull with real blood and brain tissue. We examined the performance of the detection algorithm (Oziel et al., $2020 \mathrm{~b}$ ) in this geometric and tissue configuration, which resembles more closely the clinical situation with respect to: (a) the sensitivity to small changes in the tissue/fluid volume ratio; (b) the effect of accumulated location of bleeding; (c) the difference between the response to blood injection into a balloon, which simulates the accumulation of blood in one place in the brain (such as in an extradural hematoma), in comparison to direct injection of blood into the brain tissue, such as occurs in an intraventricular hemorrhage.

\section{Materials \& Methods}

The Experimental System

As mentioned before, the system design was justified using 3D simulations (Oziel et al., 2018) and then by a simple model of gel and saline solutions representing brain and blood tissues, respectively (Oziel et al., 2020a). The experimental system constructed around an Foxfield N9923A Network Analyzer (Keysight, Santa Rosa, CA). The network analyzer was connected to the induction coil sensor by a single co-axial cable. The electrical circuit, photographs of the devices and $S_{11}$ diagram are shown in Fig. $1.1 \mathrm{~mm}$ diameter copper electric wire, used for transformer applications, was wrapped (72 loops) in a single layer around a plastic cylinder (width of $30 \mathrm{~mm}$ ). The circular cross-section of the plastic cylinder possessed a $240 \mathrm{~mm}$ diameter, $60 \mathrm{~mm}$ height and a $1 \mathrm{~mm}$ thickness. The network analyzer was connected to a laptop, Lenovo W541 (Lenovo, Morrisville, NC), through a LAN connection. The blood injection was performed by a 
119 KDS-210 Syringe pump (KD Science, Holliston, MA) that can move in 0.165 micrometer step

120

121

122

123

124

125

126

127

128

129

130

131

132

133

134

135

136

137

138

139

140

141

142

143

144

145

146

147

148

149

150

151

152

153

154

155

156

157

158

increments, equivalent to an injected volume of $0.092 \mu \mathrm{l}$ per single step. The pump was connected to the laptop via an RS232 to RJ11 cable. The laptop controls the syringe pump and collects and stores the data from the network analyzer.

\section{The Experimental Model for Simulating Internal Bleeding}

Intracranial hemorrhages are divided based on their anatomic location and can be classified into two groups: intra-axial and extra-axial ones (Caceres \& Goldstein, 2012). The intra-axial group possesses two types of hemorrhages. The first type is an intraparenchymal hemorrhage, which reflects bleeding within the brain parenchyma (Almandoz et al., 2009; Gebel et al., 1998). This type of hemorrhage has no distinctive shape and the range of blood volume is between 0 and 200 $\mathrm{ml}$, with an average volume of $68.7 \mathrm{ml}$. The second type is intraventricular hemorrhage. An intraventricular hemorrhage displays a bleeding into the brain's ventricular system (Gates et al.,1986). The range of blood volumes is in the range of 0-20 $\mathrm{ml}$ (Hallevi et al., 2010; Kramer et al., 2010). The extra-axial group consists of three types: (i) An extradural hematoma (epidural) which is a lens-shaped collection of blood between the dura and the inner surface of the skull (Pryse-Phillips, 2009), within a 0-150 ml volume range (Rivas et al.,1998); (ii) A subdural hematoma is a crescent-shaped collection of blood between the dura and the arachnoid ((PrysePhillips, 2009). The average volume of the subdural hematomas is $91 \mathrm{ml}$ in the range of 5-300 ml (Gebel et al., 1998) (iii) Subarachnoid hemorrhage is bleeding between the arachnoid membrane and the pia mater (Pryse-Phillips, 2009). The typical shape for a subarachnoid hemorrhage on CT images is a so-called 'star sign'.

In the literature, one can find different types of experimental models that simulate the brain tissue, depending on the application and the measuring instrument. For RF applications, saline solution was used at different concentrations to detect small changes in conductivity at $3.5 \mathrm{GHz}$ (Karanasiou et al., 2004). A more recent study used a gel with the dielectric properties of the brain's white matter to detect small changes in conductivity through radiometry (Groumpas et al., 2017). Nearly two decades ago a more advanced model consisting of a uniform phantom based on COST244 to create tissue-equivalent phantoms using mixtures of agar, polyethylene powder (PEP), TX-151, and sodium chloride was used to perform SAR estimates at $200-3000 \mathrm{MHz}$ frequencies (Okano et al., 2000). Using identical materials, (Velander et al., 2018) constructed a phantom to assess intraocular pressure. A good match between the dielectric parameters of the brain and the tissue of water-based agar and sugar at different doses was shown (Chew et al., 2012). A path-loss index, using a square phantom based on the dielectric parameters at a single 2.4 $\mathrm{GHz}$ frequency was measured (Roelens et al., 2006).

Using a whole animal or animal tissue for human modeling is also common in research. Lind et al. (Lind et al., 2007) presents a summary of dozens of uses of pig tissue in neurological research. Shaver et al. (Shaver et al., 1996) used 3 piglets to model brain injury after a subdural hematoma. Fantini et al. (Fantini et al., 1999) used pigs and light spectroscopy to detect disturbances in brain 
159 hemodynamics, and Zhou et al. (Zhou et al., 2009) performed similar research. Sanchez (Sanchez,

160

161

162

163

164

165

166

167

168

169

170

171

172

173

174

175

176

177

178

179

180

181

182

183

184

185

186

187

188

189

190

191

192

193

194

195

196

197

198

2013) examined in his thesis how the injection of blood into sheep brain tissue affects the signal return of an antenna in a broadband pulse. Ayati et al. (Ayati et al., 2014) researched a way to find the location of a hematoma in a sheep brain via antenna array and impedance tomography.

Since the head size and anatomy of the various in-vivo animal models differs significantly from that of the human head, it will affect the estimation of volume of blood accumulation. Therefore, we have chosen to employ in our experiment a model of human skull (Wellden, Toronto, Canada), prior to performance of clinical trials. The skull we used in the experiment is based on Medical Anatomical Human Skull model, made of PVC in a realistic adult size. The length of the skull was $17 \mathrm{~cm}$ and the width were $13.5 \mathrm{~cm}$ (on the semi minor/major axes). The volume of the brain cavity was approximately $1200 \mathrm{~cm}^{3}$ (see Fig. 2). We inserted into the skull cavity a thin plastic bag, which contained parts of defrosted calf brain (originally prepared for food). For each experiment, we verified that the volume of the brain filled the skull cavity almost completely. After inserting the brain into the skull, we filled all the remaining space with physiological saline solution. Hemorrhage was simulated by injecting defined volumes of blood into the brain tissue using a standard 2-way Foley catheter with a rubber valve (Bard, Covington, GA). The positions of catheter tips are marked in Fig. 2. We roughly divided the injection locations into two groups, relative to the center point. One injection site was at the skull center $\pm 1 \mathrm{~cm}$ (blue circles, Fig. 2) and second injection sites were at the margin of the skull (the orange circles - about $1 \mathrm{~cm}$ from the outer surface $\pm 1 \mathrm{~cm}$ ). The height of the catheter's tip was $\sim 2 \mathrm{~cm}$ from the top of the skull $\pm 1 \mathrm{~cm}$. In the experiment, we used fresh chicken blood from a chicken slaughtered for food two hours before the experiment. After an hour of experimentation, we discarded the blood (we did not use anticoagulants). The blood was injected through two routes. In the first one, the blood was injected into a rubber balloon capable of reaching a volume of $70 \mathrm{ml}$. The balloon was connected with epoxy glue to the tip of the catheter (standard catheter balloons have a volume of only $10 \mathrm{ml}$ ). The second route was a direct blood injection into the brain tissue on the same injection sites as the first route. In both cases, we used a $50 \mathrm{ml}$ syringe (MedicPro, Kuala Lumpur, Malaysia), which was controlled by a syringe pump and was connected directly to the catheter's tube.

\section{Experimental Method}

We used a network analyzer to measure $S_{21}$ - the power received in the network analyzer output port (port 2) relative to the power input port (port 1). We measured 50 equally spaced $S_{21}$ ranged from $100 \mathrm{MHz}$ and $1 \mathrm{GHz}$ per $600 \mathrm{~ms}$ with no averaging. The bandwidth was $300 \mathrm{~Hz}$ and maximum power level was $5 \mathrm{dBm}$, continuously throughout the experiment. Hemorrhage was simulated by injecting precise volumes of chicken blood into the brain tissue (with/without balloon) in $2 \mathrm{ml}$ increments. For every injected volume increment, we took $70 S_{21}$ measurements during a $1 \mathrm{~min}$ period. The injected volumes ranged from 0 to $18 \mathrm{ml}$ (9 injections).

In this experiment, we examined several parameters. As we previously described, we roughly divided the blood injection locations into those made in the center of the skull and those made at its margins. Another parameter is injection into a balloon, which intends to simulate blood

Peer) reviewing PDF | (2020:07:51224:1:2:NEW 22 Oct 2020) 
199

200

201

202

203

204

205

206

207

208

209

210

211

212

213

214

215

216

217

218

219

220

221

222

223

224

225

226

227

228

229

230

231

232

233

234

235

236

237

accumulation in a defined site in the brain, or blood injection directly into the brain tissue, which is supposed to simulate a more delocalized brain hemorrhage. Finally, to test the effect of blood pulsatility, we performed measurements in which we cyclically pushed and pulled $1 \mathrm{ml}$ of blood approximately 10 times per minute. These measurements were designed to test whether our algorithm was able to deal with periodic changes that generate noise in a different way than noise originating from an electronic source. Obviously, the heartbeat rate is much higher than the pump rate, but in our experiment the Nyquist frequency is about $30 \mathrm{~Hz}$. So high frequencies of this frequency will not be properly sampled.

To reduce RF reflection from the environment, a cylindrical Faraday cage possessing with diameter of $250 \mathrm{~mm}$ and height of $120 \mathrm{~mm}$, was placed around the inductive coil sensor. We have not maintained a fixed position of the coil and the phantom relative to the cage. So, between each measurement, the relative positions of the phantom, the cage, and the coil have slightly changed. We estimated the internal errors, such as thermal noise and internal drift in the same method as in the previous experiments (Oziel et al., 2020a): 20 minutes of the $S_{21}$ measurements under 3 different scenarios: (a) The device was turned on for half an hour; (b) when the device was turned on for two hours (hot device); and (c) in the presence and absence of the skull with the brain. Three repeats were done for each scenario. After verifying that the noise distribution was normal, we calculated the average over the different measurements and the standard deviation for both the amplitude and phase. We could estimate the relative error due to noise was $2.1 \cdot 10^{-4} d B$ in the amplitude measurements and $1 \cdot 10^{-3}$ degree in the phase measurements.

Changes in the relative position of the faraday cage during measurement have a strong effect on the electrical signal, which is fundamentally different from changes resulting from the injection of blood into the skull, which is expressed in the form of high and narrow peaks over the entire frequency range. Measurements in which a relative change in the location of the cage was detected were rejected.

\section{Algorithm}

The description of the algorithm used for the analysis of the results is described as follows:

1. Let us define $t_{\text {start }}$ and $t_{\text {end }}$ as the start and the end time points at which the phase and amplitude are measured across the RF coil. In our experiment there is actually a correlation between time and the injected volume, since we injected in increments of $2 \mathrm{ml}$ of blood volume, every single minute. However, the algorithm described here is general.

2. Let us define $F_{0}$ as the analyzed frequency range, where $\mathrm{n}$ is the number of frequencies sampled in the $F_{0}$ frequency range. In our experiment, $F_{0}$ is in range of $100-1000 \mathrm{MHz}$ and $\mathrm{n}=50$. In general, we require that $n \geq 30$.

3. The Network analyzer was used to record the amplitude, $A$, and the phase, $\varnothing$, at each frequency $f_{0} \in F_{0}$, during the period of time from $t_{\text {start }}$ to $t_{\text {end }}$ (to avoid repetition, we will use the notation $\mathrm{E}$, as a place holder for amplitude and phase). For each frequency, $f_{0}$, and time, $t$, we 
255

256

257

258

259

260

261

262

263

264

265

266

267

268

270

271

272

$269 \quad \overline{R(F)}=\frac{\sum \mathrm{R}(\mathrm{F})}{n}$

measure multiple values of $\mathrm{E}$ (70 samples in our experiments) in a predetermined narrow time window, $\Delta t$, to increase the signal to noise ratio (SNR) and calculate the average, avg $\left(E\left(f_{0}, t\right)\right)$, and standard deviation, $\operatorname{std}\left(E\left(f_{0}, t\right)\right)$ for that time, $t$. Again, in this experiment $\Delta t$, corresponds to the one-minute time duration between each injected volume of blood. As before, the algorithm developed here is general and not related to the measurement time window.

4. The algorithm is based on measuring the changes in measurements of $E\left(f_{0}, t\right)$ between those made at $t_{\text {start }}$ and $t_{\text {end }}$.

5. As a first step, we filter out from all the measurement frequencies, all frequencies at which $\left|\operatorname{avg}\left(E\left(f_{0}, t_{\text {end }}\right)\right)-\operatorname{avg}\left(E\left(f_{0}, t_{\text {start }}\right)\right)\right|<$ Measurements error $\left(2.1 \cdot 10^{-4} d B\right.$ for the amplitude and $1 \cdot 10^{-3}$ degree for the phase) and define $F_{1}$ as the set of all frequencies in the measured range in which the change is higher than the measurement error.

6. Let us define the ratio between the change in the average values at the two time points and the noise (Represented by the standard deviation of E) at each frequency, $f_{1} \in F_{1}$ defined in step 5., as follows:

$$
R\left(f_{1}\right)=\frac{\left|\operatorname{avg}\left(E\left(f_{1}, t_{\text {end }}\right)\right)-\operatorname{avg}\left(E\left(f_{1}, t_{\text {start }}\right)\right)\right|}{\max \left(\operatorname{std}\left(E\left(f_{1}, t_{\text {end }}\right)\right), \operatorname{std}\left(E\left(f_{1}, t_{\text {start }}\right)\right)\right)}
$$

Where E (as defined in paragraph 3) can be the amplitude or the phase.

7. The next step is to filter out all frequencies in which $R\left(f_{1}\right)<2$ and let's define $F_{2}$ as the set of all frequencies in the measured range at which $R\left(f_{1}\right) \geq 2$ (A dimensionless number). This should remove most of the noise-induced artifacts.

8. Then, for $f_{2} \in F_{2}$ we examine whether the difference between $E\left(f_{2}, t_{\text {end }}\right)$ and $E\left(f_{2}, t_{\text {start }}\right)$ is statistically significant for each of the frequencies in the set $F_{2}$. To this end, we use the twosample Kolmogorov-Smirnov test (Lilliefors, 1967), which examines whether the two groups, $E\left(f_{2}, t_{\text {end }}\right)$ and $E\left(f_{2}, t_{\text {start }}\right)$, belong to the same distribution. We then define the set of frequencies, $F$, in which the measurements satisfy the following properties: For each set of the measurements $\mathrm{E}$ in the set of frequencies, $\mathrm{F}, R\left(f_{2}\right) \geq 2$ and the statistical test shows that there is a statistically significant difference between $E\left(f, t_{\text {end }}\right)$ and $E\left(f, t_{\text {start }}\right)$ where $f \in F$.

9. Finally, let us define $\overline{R(F)}$ as:

Here also, $\overline{R(F)}$ is a dimensionless number and is the average change in the measured amplitude or phase between two measurements across a time interval relative, to the standard deviation of the experimental measurements for all the statistically meaningful frequencies. Importantly, we 
273 divide the contribution of $\mathrm{R}(\mathrm{F})$ from all frequencies by the number of frequencies of $f_{0}$. Doing

274 this, allows us to deal with edge cases where the difference between $E\left(f, t_{\text {end }}\right)$ and $E\left(f, t_{\text {start }}\right)$ can

275 be a consequence of noise from a small number of frequencies.

276 In the next paragraph, we will show that the value of $\overline{R(F)}$ is a very robust and accurate parameter 277 that can serve as a marker for changes in blood volume. This can serve as a very simple indication 278 of changes in the medical condition of the patient. It must be emphasized that this study was done 279 employing a brain tissue model in a skull, and the algorithm must be clinically verified in the 280 future.

281

282

283

284

285

286

287

288

289

290

291

292

293

294

295

296

297

298

299

300

301

302

303

304

305

306

307

308

309

310

311

312

\section{Results}

We have obtained a complete record of the changes in amplitude and phase measured across the $\mathrm{RF}$ coil in response to injection of blood in our experimental model of intracranial hemorrhages, throughout the frequency range of $100 \mathrm{MHz}$ to $1 \mathrm{GHz}$. The results are qualitatively similar to those presented in our previous theoretical studies (Oziel et al., 2017; Oziel et al., 2018). We find that the change in amplitude and phase across the RF coil upon an injection of blood, is a complex function of frequency, of the injected blood volume and of the location. The frequency response to the injected blood is neither uniform across all frequencies nor monotonous with the increase in the injected blood volume. In fact, at some frequencies there are no changes with the injected blood volume, while at others the changes are substantial. Figure 3 illustrates the complexity of the frequency response to blood injection into the skull's center. The frequencies in this figure were chosen at random, just for demonstration purposes. Figure 3 shows the average changes in amplitude and phase due to nine injections of consecutive $2 \mathrm{ml}$ volumes of blood. The left panel shows the change in the absolute value of the amplitude and the right panel shows the change in the absolute value of the phase. The abscissa is the volume of the injected blood, the blue squares markers are the average measurement values of $\mathrm{E}$ for $559.18 \mathrm{MHz}$, the red circles markers are the measurement values of $\mathrm{E}$ for $540.82 \mathrm{MHz}$, and the magenta asterisk markers are the measurement values of $\mathrm{E}$ for $173.47 \mathrm{MHz}$.

It is evident from Fig. 3 that the magnitudes and nature of the change in amplitude and phase between consecutive injections of $2 \mathrm{ml}$ volumes, are dependent on the frequency at which the measurements were made. For example, at $559.18 \mathrm{MHz}$ and $540.82 \mathrm{MHz}$, the difference between the values of amplitudes and phase for consecutive injections of $2 \mathrm{ml}$ volumes can be substantial. In contrast, at $173.47 \mathrm{MHz}$, the amplitude and phase do not vary significantly between consecutive injections. The frequencies of $559.18 \mathrm{MHz}$ and $540.82 \mathrm{MHz}$ are close to each other relative to the entire range of frequencies in this experiment. However, the response to the injection of $2 \mathrm{ml}$ of blood is substantially different. The right panel shows that up to a total injected volume of $12 \mathrm{ml}$, the curve representing change of phase increases monotonically with the injection of consecutive volumes of $2 \mathrm{ml}$ blood, for both frequencies, of $559.18 \mathrm{MHz}$ and of $540.82 \mathrm{MHz}$. However, the magnitude of the change of phase remains constant for further injections of blood, for the frequency of $559.18 \mathrm{MHz}$, but not for the frequency of $540.82 \mathrm{MHz}$. In contrast, the change in 
313 amplitude due to injection of $2 \mathrm{ml}$ of blood exhibits a completely different pattern for these 314 frequencies. At the frequency of 559.18, the value of the amplitude across the RF coil increases 315 monotonically with the injected volume of blood. However, at a frequency of $540.82 \mathrm{MHz}$, the 316 amplitude across the RF coil increases with an increase in the injected volume of blood, until an 317 injected volume of $10 \mathrm{ml}$. At this frequency, the amplitude begins to dramatically decrease with 318 any further injection of blood after a total volume of $10 \mathrm{ml}$ blood was injected.

319 In summary, this example demonstrates that there is no trivial correlation between changes in the

320

321

322

323

324

325

326

327

328

329

330

331

332

333

334

335

336

337

338

339

340

341

342

343

344

345

346

347

348

349

350

351 volume of blood injected into the brain and the phase and amplitude measurements across the RF coil. Obviously, changes in blood volume cause changes in the amplitude and frequency across the single coil RF. However, these changes are not trivial. For this technology to have practical value, there is a need for an algorithm that can analyze the entire frequency range and extract the appropriate information. We have introduced the algorithm in the Material and Methods section and will subject the experimental results in this study to this algorithm.

Histograms of all $R(F)$ values in this experiment, for amplitude and phase, are shown on the lefthand side of Fig. 4. This kind of distribution can be transformed into a normal distribution by applying a $\log _{10}$ transformation (Howell, 2007). The $\log _{10}(\overline{R(F)})$ values for amplitude and phase are shown on the right-hand side panels of Fig 4 . The center of the normal distribution for the amplitude is around $\overline{R(F)}=15$. For the phase, there are extreme values for $\overline{R(F)},(\overline{R(F)}$ $>100$ ). After filtering out these extreme values (the criteria used to filter out the extreme values will be presented in the discussion section), we obtain a normal distribution where the center of the distribution for the phase is around $\overline{R(F)}=11$.

The processed raw data from the experiments is given on Fig. 5. The figure shows the $\log _{10}$ $(\overline{R(F)})$ values for amplitude and phase, for each experimental condition, that includes variation in volume of injected blood and location of the injected volume.

Figure 5, show the $\log _{10}(\overline{R(F)})$ values for amplitude and phase at a boxplot format, where the abscissa is the volume of injected blood. For example, the values at $4 \mathrm{ml}$ represent the algorithm values for the difference between 0 and $4 \mathrm{ml}$, as well as the algorithm values for the difference between 2 and $6 \mathrm{ml}, 4$ and $8 \mathrm{ml}$, and so on. Each data point represents an experiment. The red line inside the blue box represent the median of each set of measurements and the blue box represent the interquartile range (25th to the 75 th percentile). This graph shows the following phenomena. In general, the change for the amplitude is between the signal-to-noise ratio of $3\left(\log _{10}(\overline{R(F)})\right.$ $\sim 0.5$ ) for low differences in the accumulated blood volume, to $15-50$ for high differences between the accumulated blood volume, $\left(\log _{10}(\overline{R(F)}) \sim 1.7\right)$. The median values of the amplitude are lower than the phase values. However, the phase values include far more extreme values (a signalto-noise ratio of 500-1000) As expected, the median value increases depending on the injection volume up to around 14-18 $\mathrm{ml}$. It appears that the effect of amplitude or phase for a $14 \mathrm{ml}$ change in blood volume is not significantly different from the change in amplitude or phase for 16 or 18 $\mathrm{ml}$. 
352

353

354

355

356

357

358

359

360

361

362

363

364

365

366

367

368

369

370

371

372

373

374

375

376

377

378

379

380

381

382

383

384

385

386

387

388

389

390

391

Figure 6 shows the results of all the various experiments on a consolidated graph. The abscissa is $\log _{10}(\overline{R(F)})$ for the amplitude, the ordinate is $\log _{10}(\overline{R(F)})$ for the phase. The different markers represent blood injection volumes in the $2-18 \mathrm{ml}$ range, and the colors represent the different experimental groups.

The green markers are for an experiment in which $1 \mathrm{ml}$ blood was pushed into and out of the balloon in the brain tissue at a frequency of $0.17 \mathrm{~Hz}$ at the center of the skull. The group of experiments in which the blood was injected into a balloon (both center and margin) in increments of $2 \mathrm{ml}$, is marked by blue. It is interesting to notice, that in most of the experiments there is a linear relationship for $\log _{10}(\overline{R(F)})$ between amplitude values and phase values. However, in a small fraction of the results, (5.2\%) the change in phase is 10 times as large as the change in the amplitude. This phenomenon can be explained by the convergence of different boundary conditions, so that local enhancement is created for a specific injection. (In the next paragraph, we will discuss the algorithm for estimating the volume of blood flow, where we will see a model that ignores these values. It is important to understand that these high values are strong evidence of an increase in blood volume in the brain, and from a clinical point of view, they are a trigger to alert of a significant change in blood volume in the brain). The correlation between $\log _{10}(\overline{R(F)})$ is the same as that for blood injected in a balloon at both center and the margin sites. Again, in a small fraction of the measurements the change in phase values are larger than the change in amplitude values.

\section{Discussion}

Our study demonstrates that the monotonic increase in blood volume in the skull does not lead to a monotonic increase in the measured amplitude and phase change across the coil. We also observe that there are a small number of frequencies that are strongly affected by the change in the tissue/fluid volume ratio in the brain. However, the intensity of the change is different from frequency to frequency and from experiment to experiment. This uncertainty about which of the frequencies will produce a stronger response to changes in blood volume in the head and the lack of monotonicity in the response at many frequencies have suggested to us that the measurements should be made across a large range of frequencies, in the hope that this will capture the frequencies with a strong response. In this study we made the measurements across a range of frequencies from (100-1000 MHz), although there is no technological impediment to making measurements across a larger range.

In the paragraph describing the algorithm, we define $\overline{R(F)}$ as the sum of the algorithm values, divided by the number of $f_{0}$ values - the number of frequencies scanned. This change allows us to give less weight to cases where, at a single frequency, there is a significant power change as a result of random noise (e.g. spike noise).

Peer) reviewing PDF | (2020:07:51224:1:2:NEW 22 Oct 2020) 
392 One can see in Fig. 6 that all the algorithm values (green markers) which arrived from the $1 \mathrm{ml}$ 393 group, have values less than 0.5 , namely $\overline{R(F)}_{\text {amp/phase }}<3$. This is in agreement with our 394 previous article (Oziel et al., 2020b), in which we showed that this threshold value corresponds to 395 a value that distinguishes small changes of noise and volume of blood from real significant changes 396 of the tissue/fluid volume ratio in the head. A change in the blood/tissue ratio of $P\left(\log _{10}(\overline{R(F)})\right)$ $397=0.5$, which equals to $\overline{R(F)}=3$ can be used as a measure for the occurrence (a diagnostic index) 398 of a clinical change in the blood/tissue volume ratio.

399

400

401

402

403

404

405

406

407

408

409

410

411

412

413

414

415

416

417 We used a linear regression model with two predictors $\log _{10}(\overline{R(F)})$ (for amplitude and phase) as

418 follows:

419

420

421

422

423

424

425

426

427

428

429

Correctly estimating the volume of blood that accumulates in the brain is of clinical importance. We would like to estimate the volume of the injection depending on the values of $\mathrm{R}(\mathrm{F})$, amplitude and phase. For this purpose, we selected the set of values that best described most of the measurements. As mentioned in the previous sections, when we remove the cases in which the phase had extreme values, there is a linear dependence between the values of amplitude and phase. Therefore, we have chosen the measurements that maintain:

$$
0.8<\frac{\log _{10}\left(\overline{R(F)_{\text {phase }}}\right)}{\log _{10}\left(\overline{R(F)_{\text {amp }}}\right)}<1.5
$$

At the same time, values higher than the noise values were selected:

$$
\log _{10}\left(\overline{R(F)_{\text {amp,phase }}}\right)>0.5
$$

To generate the model, we selected all the measurements that meet the above criteria from the measurement sets presented in Fig. 5 and 6, and we filter out each injected volume belonging to the outlier's values from the box plots of Fig. 5 (marked with a red plus). We would like to do so, to avoid creating a situation in which the estimated blood volume values will be strongly influenced by the extreme values.

$$
Y=b_{0}+b_{1} X_{1}+b_{2} X_{2}+b_{3} X_{1}^{2}+b_{4} X_{2}^{2}
$$

Figure 7 visually presents the adjustment of the chosen measurement values to the linear regression model where the mean square error is $1.76 \mathrm{ml}$.

To examine the model, we ran eight additional measurements (including 9 injections from 2-18 $\mathrm{ml})$ that were not part of the measurement set for model construction. The measurements for the model of the brain were different than the measurements made for the model creation, by the following parameters: We replaced the brain tissue 3 times. Since the brain structure is not uniform, the brain volume and the spatial structure introduced to the skull were slightly different for each replacement of brain tissue and as well the amount of the saline. We verified that the 
430 locations of blood injection would not be in the same places as in the model building phase. We 431 made sure as well, to change the phantom position relative to the coil between each measurement 432 (around half a cm for each direction) and we also took care to remove and return the coil between 433 each measurement, so that its position will somewhat vary. Four measurements were performed 434 when the blood was injected into a balloon, and the other four measurements were made following 435 direct injection into the brain tissue. For each group of four measurements, two measurements 436 were made close to the center of the brain and two closes to the phantom margin but not in the 437 same places where the injection sites for model creation were performed.

438 As previously mentioned, the basis for deciding whether there has been a significant change in 439 tissue/blood volume ratio was that $\log _{10}\left(\overline{R(F)_{\text {amp,phase }}}\right)>0.5$. For a $2 \mathrm{ml}$ volume difference, in $44082 \%$ of the tests the algorithm values were higher than the threshold; for a $4 \mathrm{ml}$ volume differences,

441

442

443

444

445

446

447

448

449

450

451

452

453

454

455

456

457

458

459

460

461

462

463

464

465

466

467

468

469 about $90 \%$ of the measured values were higher than the threshold. At $6 \mathrm{ml}$, approximately $99 \%$ measured values were higher than the threshold, and from 8 to $18 \mathrm{ml}, 100 \%$ of the values were higher than the threshold.

Figure 8 shows the mean and standard deviation of the estimated blood volumes after filtering out the values that do not satisfy Equations 3 and 4. One should note that the estimated volumes are greater than the true volume values for all cases. This phenomenon is understandable considering that most of the extreme values that were filtered out during model construction were high, and therefore, the volume estimation is biased upward. One can see how the error is more or less fixed ( $2.3 \mathrm{ml}$ average for the average volumes) and a monotonous increase of the estimated volume in the $0-16 \mathrm{ml}$ range. One way to interpret the figure is to evaluate what is the probability to estimate a volume $\mathrm{Y}$ from the analysis algorithm if volume $\mathrm{X}$ was actually injected. For example, when an actual injection of $12 \mathrm{ml}$ blood takes place, the probability that the analysis algorithm will estimate a value between 10 to $16 \mathrm{ml}$ is about $50 \%$. However, the probability that the detection algorithm will estimate that the injection is between 10 to $16 \mathrm{ml}$ if the actual injection is $2 \mathrm{ml}$ is almost zero.

\section{The Phantom as a Human Head Model}

Designing a human head model for the simulation of head blood flow should take into account a number of parameters, both in the area of the dielectric properties of the various head components and in the different physiological aspects.

Regarding the dielectric properties of the phantom, even though chicken red blood cells differ from mammalian blood cells both in their shape and the existence of a cell nucleus in avian blood cells (Zentgraf et al., 1969), the dielectric properties of hen blood are similar to those of human blood in the frequency range of 100-1000 MHz. Table 1 presents a comparison between the dielectric parameters of human blood and chicken blood, as well as dielectric data for calf and human brain. At the beginning of each experiment (i.e. before the first blood injection), the phantom contained no blood at all - unlike a human head. However, we believe that this change doesn't present a major impact on the experimental result since the empty phantom parts was filled with physiological saline. It can be seen in Table 1 that the dielectric values of the physiological saline are very close to that of blood. Similarly, it can be seen in Table 1 that the electrical properties of 
470 a human skull and of PVC are also very close. Since the head's skin thickness is very small (about $4713 \mathrm{~mm}$ ), and in addition, the electrical properties of human skin are very close to those of the Bovine 472 brain - the effect of the skin layers on the signal strength is nil and can be neglected.

473 Another physiological aspect is the constant volume of the skull, due to the skull rigidity. This 474 aspect is also preserved in our experiments. When we have injected the blood, the pressure in the 475 skull has raised and in most of the cases, the brain tissue was slightly compressed towards the 476 small openings at the base of the skull (The physiological term for this process is called 477 Herniation). The volume of CSF (Cerebrospinal fluid) in the head varies over time depending on 478 a large number of variables such as the blood pressure, posture change, and etc. (Van Beek et al., 479 2008) However, experiments in rabbits (Zhao et al., 2019) in which the change in the signal phase 480 was monitored for 24 hours, showed that the accumulation of blood in the brain has a contribution 481 to the change in the phase 10 times greater than the contribution in the phase as a result of the 482 normal changes in fluid in the head.

483 Brain activity produces electrical signals (EEG). However, the frequency range of the electrical 484 activity in the brain is much lower $(1-2000 \mathrm{~Hz})$ (Moffett et al., 2017) than the operating range of 485 the system proposed in this paper $(100-1000 \mathrm{MHz})$. Therefore, the electrical signals from the brain 486 activity do not actually affect the signal transmitted/received from the measurement system.

487 An additional physiological aspect is blood pulsatility in the head. This aspect was examined and 488 reported in the results section, where we tested (separately) the signal strength resulting from an 489 injection of $1 \mathrm{ml}$ of blood in and out the phantom. We have verified that this signal is very low and 490 is below the algorithm threshold of detecting a significant change in blood volume in the head.

491

492

493

494

495

496

497

498

499

500

501

502

503

504

505

506

507

508

509

\section{Limitation of the study}

The study assumes that the measurement setup monitors the patient while the hemorrhage is developing. In order to bring the research into clinical trial phase, two significant problems should be resolved. These two problems are deeply related to the capabilities of the system to deal with motion artifacts.

The first topic is to find a better alternative to the Faraday cage we have used so far. In the experimental system, we used a metallic cylinder similar to a pot to avoid interference resulting from the reflection of RF waves from the environment.

This pot has a number of drawbacks. The first is the patient's discomfort as a result of attaching a massive metal block to the head. The second is the difficulty of bringing back the pot to its exact location when the coil is temporarily removed. One possible solution is the use of a mesh mask similar to those used for radiotherapy. The use of such a mask allows the coil to be positioned back to its exact location, which is convenient both for the patient and caregiver, and the configuration of this coil would reduce the patient's discomfort.

However, the mesh also has a drawback common to that of the pot. This disadvantage is the closed spatial structure, which returns the RF reflections from the boundaries back to the coil. This configuration is similar to the configuration of the two coils, in terms of the sensitivity of the 
510 system to coil displacements or the head displacements with respect to the coil. A possible solution 511 would be to build a new coil, where the external coil is covered with a metallic material. This 512 configuration will significantly reduce the waves transmitted to the area outside the coil and 513 therefore significantly reduce the reflections from the environment, although it will not prevent it 514 altogether.

515 The second point is the need to build a cable-free measurement system. In such a futuristic setup, 516 the RF components should be physically connected as tightly and as close as possible to the coil, 517 with no flexible cables between the coil and transmitter/receiver. Similarly, the sampling 518 component should be designed to transmit the amplitude/phase values to a computer or other 519 communication component, but will not be connected by a communication cable. These 520 enhancements will allow maximum comfort for the patient and significantly reduce noise insertion 521 as a result of unavoidable patient movement in the space.

522 Solving these two problems is expected to lead to the ability to overcome both motion artifacts

523 arising from unavoidable movements of the patient as well as motion artifacts arising from the 524 wearing and removal of the measurement system.

525 Although these two problems are very significant for the general use of this technology, it is 526 important to note (Davis et al., 2006) that in many cases (over 70\%) the blood volume in the brain 527 increases by 3 hours after arrival to the hospital (Davis et al., 2006). Therefore, if the hospital has 528 the ability to stabilize the patient and prevent motion artifacts, clinical uses of this technology can 529 be seen even before upgrading the present set-up.

530

531 Cost

532 A new Network Analyzer can indeed cost several thousand dollars. However relative to CT or 533 MRI which cost millions of dollars, such a solution is relatively inexpensive. In addition, the 534 authors of this article believe that manufacturing such a device in an industrial manner so that it 535 measures a limited and constant number of frequencies can significantly lower the price of such a 536 device.

537

538

\section{Conclusions}

540 The current study validates the algorithm accuracy based on using a phantom that has similar 541 dielectric properties and spatial structure as those possessed by a human head. We have shown in 542 this study that the suggested simple and inexpensive measurement system combined with the 543 algorithm can detect slight changes in the tissue/fluid volume ratio in the head, up with the ability 544 to detect progressive internal bleeding in the head of a minimal volume of $2 \mathrm{ml}$. Based on such 545 characteristics, it may be possible to build a simple device capable of detecting and alerting, at the

546 beginning of the increase in the volume of bleeding in the head, which may help physicians initiate 547 early intervention to prevent further deterioration of brain injury. We also showed how the volume 548 of blood injected into the brain can be roughly measured. However, it is clear that there is a need 549 to improve and to test the algorithm prior to clinical trials. 
550

551

552

553

554

555

556

557

558

559

560

561

562

563

564

565

566

567

568

569

570

571

572

573

574

575

576

577

578

579

580

581

582

583

584

585

586

587

\section{Acknowledgements}

This work is based on a portion of a dissertation to be submitted by Moshe Oziel in partial fulfilment of the requirements for a PhD degree to Tel-Aviv University.

\section{References}

Alanen E., "Penetration of electromagnetic fields of an open-ended coaxial probe between $1 \mathrm{MHz}$ and $1 \mathrm{GHz}$ in dielectric skin measurements," in Physics in Medicine \& Biology, vol. 47, 1999, p. 169.

Almandoz J. D., Schaefer P. W., Forero N. P., Falla J. R., Gonzalez R. G., \& Romero J. M., "Diagnostic accuracy and yield of multidetector CT angiography in the evaluation of spontaneous intraparenchymal cerebral hemorrhage," in American Journal of Neuroradiology, Vol 30, 2009, pp. 1213-1221.

An S. J., Kim T. J., \& Yoon B. W., "Epidemiology, risk factors, and clinical features of intracerebral hemorrhage: an update," in Journal of stroke, Vol 19, 2017, pp. 3-3.

Ayati S. B., Bouazza-Marouf K., Kerr D., "Hematoma detection using EIT in a sheep model," in Taylor \& Francis, International Journal of Computers and Applications, Vol. 36, 2014, pp. 87-92. Brain Aneurysm Foundation. Brain Aneurysm Statistics and Facts. [Online] 2019. Available: https://www.bafound.org/about-brain-aneurysms/brain-aneurysm-basics/brain-aneurysmstatistics-and-facts/.

Brott T., Broderick J., Kothari R., Barsan W., Tomsick T., Sauerbeck L., "Early hemorrhage growth in patients with intracerebral hemorrhage," in Stroke, Vol 28, 1997, pp. 1-5.

Caceres J. A. and Goldstein J. N. "Intracranial hemorrhage," in Emergency medicine clinics of North America, Vol 30, 2012, p 771.

Chew K. M., Sudirman R., Seman N., \& Yong C. Y., "Human Brain Phantom Modeling Based on Relative Permittivity Dielectric Properties," in 2012 International Conference on Biomedical Engineering and Biotechnology, IEEE, 2012, pp. 817-820

Chung K. T., Sabo A., \& Pica A. P., "Electrical permittivity and conductivity of carbon black-polyvinyl chloride composites" in Journal of Applied Physics, Vol 53, 1982, pp. 6867-6879. Cleveland Clinic. Cleveland Clinic's 16th annual Medical Innovation Summit. [online] 2019. Available: https://consultqd.clevelandclinic.org/ cleveland-clinic-unveils-top-10-medicalinnovations-for-2019/

Davis S. M., Broderick J., Hennerici M., Brun N. C., Diringer M. N., Mayer S. A., "Hematoma growth is a determinant of mortality and poor outcome after intracerebral hemorrhage," in Neurology, Vol 66, 2006, pp. 1175-1181. 
588 Dowlatshahi D., "Defining hematoma expansion in intracerebral hemorrhage relationship with

589

590

591

592

593

594

595

596

597

598

599

600

601

602

603

604

605

606

607

608

609

610

611

612

613

614

615

616

617

618

619

620

621

622

623

624

625

patient outcomes," in Neurology, vol. 76, 2011, pp. 1238- 1244.

Fantini S., Hueber D., Franceschini M. A., Gratton E., Rosenfeld, W. Stubblefield P. G., "Noninvasive optical monitoring of the newborn piglet brain using continuous-wave and frequencydomain spectroscopy in IOP Publishing, Physics in Medicine and Biology, Vol. 44, 1999, pp. 1543-1543.

Gabriel S., Lau R. W., \& Gabriel C., "The dielectric properties of biological tissues: II. Measurements in the frequency range $10 \mathrm{~Hz}$ to $20 \mathrm{GHz}$," in Physics in medicine \& biology, Vol. 41, 1996, p. 2251.

Gates P. C., Barnett H. J., Vinters H. V., Simonsen R. L., \& Siu K. E., "Primary Intraventricular Hemorrhage in Adults," in Stroke, Vol. 17, 1986, pp. 872-877.

Gebel J. M., Sila C. A., Sloan M. A., Granger C. B., Weisenberger J. P., Green C. L., \& Mahaffey $\mathrm{K}$. W., "Comparison of the $\mathrm{ABC} / 2$ estimation technique to computer-assisted volumetric analysis of intraparenchymal and subdural hematomas complicating the GUSTO-1 trial," in Stroke, Vol 29, 1998, pp. 1799-1801.

González C. A. and Rubinsky B., "Frequency dependence of phase shift in edema: a theoretical study with magnetic induction," in IEEE Engineering in Medicine and Biology 27th Annual Conference, 2006, pp. 3518-3521

González C. A, Blumrosen, G., \& Rubinsky, B., "Remote Monitoring of Internal Bleeding Based on Magnetic Induction and Cellular Phone Technology: A Potential Application in Poor Regions in México," in Revista Computación y Sistemas, Vol 14, 2010, pp. 187-195

Gonzalez C. A., Valencia J. A., Mora A., Gonzalez F., Velasco B., Porras M. A. \& Rubinsky B., "Volumetric electromagnetic phase-shift spectroscopy of brain edema and hematoma," in Plos one, Vol 8, 2014, [online] https://journals.plos.org/plosone/article?id=10.1371/journal. pone. 0063223

Griffths H., Stewart W. R., \& Gough W., "Magnetic induction tomography: a measuring system for biological tissues," in Annals of The New York Academy of Sciences, Vol. 873, 1999, pp. 335345

Groumpas E., Koutsoupidou M., Uzunoglu N., \& Karanasiou I. S., "Sensing local temperature and conductivity changes in a brain phantom using near-field microwave radiometry," in IEEE, Antenna Technology: Small Antennas, Innovative Structures, and Applications (iWAT), 2017 International Workshop on, pp. 293-295.

Hallevi H., Dar, N. S. Barreto A. D., Morales M. M., Martin-Schild S., Abraham, A. T. \& Savitz, S. I., " The IVH score: a novel tool for estimating intraventricular hemorrhage volume: clinical and research implications," in Critical care medicine, Vol. 37, 2009, p. 969.

Hasgall P.A., Di Gennaro F., Baumgartner C., Neufeld E., Lloyd B., Gosselin M. C., Payne D., Klingenböck A., Kuster N., "T'IS Database for thermal and electromagnetic parameters of biological tissues" Version 4.0. [Online] May 15, 2018. www. itis.ethz. ch/database. 
626 Haynes M., Stan, J., \& Moghaddam M., "Real-time microwave imaging of differential temperature 627 for thermal therapy monitoring," in IEEE Transactions on Biomedical Engineering, Vol. 61, 2014, 628 pp. 1787-1797

629 Howell D. C., Statistical methods for psychology (6th ed), Belmont, CA: Thomson Wadsworth, 6302007.

631 Huisman T. A., "Intracranial hemorrhage: ultrasound, CT and MRI findings," in European 632 radiology, Vol 15, 2005, pp. 1238-1244

633 Karanasiou I. S. \& Uzunoglu, N. K., "Experimental study of 3D contactless conductivity detection 634 using microwave radiometry: A possible method for investigation of brain conductivity 635 fluctuations," in Engineering in Medicine and Biology Society, IEMBS'04, 26th Annual 636 International Conference of the IEEE, 2004, Vol. 1, pp. 2303-2306.

637 Kellner C. P., Sauvageau E., Snyder K. V., Fargen K. M., Arthur, A. S. Turner, R. D. \& 638 Alexandrov A. V., "The VITAL study and overall pooled analysis with the VIPS non-invasive 639 stroke detection device," in Journal of neurointerventional surgery, Vol 10, 2018, pp. 1079-1084

640 Kramer A. H., Mikolaenko I., Deis N., Dumont A. S., Kassell N. F., Bleck T. P., \& Nathan B. A., 641 "Intraventricular Hemorrhage Volume Predicts Poor Outcomes but Not Delayed Ischemic 642 Neurological Deficits Among Patients with Ruptured Cerebral Aneurysms," in Neurosurgery, Vol. 643 67, 2010, pp. 1044-1053

644 Kratzenberg E., Afsar M. N., \& Wang, Y., "Complex permittivity measurements of chicken 645 blood," in Microwave and optical Technology letters, Vol. 39, 2003, pp. 54-56.

646 Lilliefors H. W., "On the Kolmogorov-Smirnov test for normality with mean and variance 647 unknown," in Journal of the American statistical Association, vol. 62, 1967, pp. 399--402.

648 Lind N. M., Moustgaard A., Jelsing J., Vajta G., Cumming P., \& Hansen A. K., "The use of pigs 649 in neuroscience: modeling brain disorders," in Elsevier, Neuroscience and Biobehavioral Reviews, 650 Vol. 31, 2007, pp. 728-751

651 Little R. D., "Increased intracranial pressure," in Clinical Pediatric Emergency Medicine,, Vol 9, 652 2008, pp. 83-88

653 Mobashsher A. T. and Abbosh A. M., "Three-dimensional human head phantom with realistic 654 electrical properties and anatomy," in IEEE Antennas and Wireless Propagation Letters, Vol. 13, 655 2014, pp. 1401-1404.

656 Moffett S. X., O’Malley S. M., Man S., Hong D., \& Martin J. V., "Dynamics of high frequency 657 brain activity." In Scientific reports, Vol 7, 2017, pp. 1-5.

658 Okano Y., Ito K., Ida I., \& Takahashi M., "The SAR evaluation method by a combination of 659 thermographic experiments and biological tissue-equivalent phantoms," in IEEE Transactions on 660 Microwave Theory and Techniques, Vol. 48, 2000, pp. 2094-2103

661 Oziel M., Korenstein R., \& Rubinsky B., "A Brain Phantom Study of a Noncontact Single 662 Inductive Coil Device and the Attendant Algorithm for First Stage Diagnosis of Internal Bleeding 663 in the Head," in Journal of Medical Devices, Vol 14, 2020, pp. 1-11. 
664 Oziel M., Hjouj M., Rubinsky, B. \& Korenstein R., "Multifrequency Analysis of Single Inductive 665 Coil Measurements Across a Gel Phantom Simulation of Internal Bleeding in the Brain," in 666 Bioelectromagnetics., Vol 41, 2020, pp. 21-33.

667 Oziel M., Korenstein R., \& Rubinsky B., "Non-Contact Monitoring of Temporal Volume Changes 668 of a Hematoma in the Head by a Single Inductive Coil: A Numerical Study," in IEEE Transactions 669 on Biomedical Engineering, Vol 66, 2018, pp. 1328-1336

670 Oziel M., Hjouj M., Gonzalez C. A., Lavee J., \& Rubinsky B. "Non-ionizing radiofrequency 671 electromagnetic waves traversing the head can be used to detect cerebrovascular autoregulation 672 responses," in Scientific reports, Vol 6, 2016, [online] https:/www.nature.com/articles/srep21667

673 Oziel M., Korenstein R., \& Rubinsky B., "Radar based technology for non-contact monitoring of 674 accumulation of blood in the head: A numerical study" in PloS one, Vol 12, 2017, [online] 675 https://journals.plos.org/plosone/article?id=10.1371/ journal.pone.0186381

676 Pryse-Phillips W., "Companion to Clinical Neurology," in Oxford University Press, 2009.

677 Qureshi A. I., Mendelow A. D., \& Hanley D. F., “Intracerebral hemorrhage,” in The Lancet, Vol 678 373, 2009, pp. 1623-1644.

679 Munawar Qureshi A., Mustansar Z., \& Mustafa S., "Finite-element analysis of microwave 680 scattering from a three-dimensional human head model for brain stroke detection," in Royal 681 Society open science, Vol 5, 2018, [online] https:

682 //royalsocietypublishing.org/doi/full/10.1098/rsos.180319

683 Rao C. V. P., "Real-time noninvasive monitoring of intracranial fluid shifts during dialysis using 684 volumetric integral phase-shift spectroscopy (VIPS): A proof-of-concept study," in Neurocritical 685 care, Vol 28, 2018, pp. 117-126

686 Richer A. and Adler A., "Eddy current based flexible sensor for contactless measurement of 687 breathing", In 2005 IEEE Instrumentational and Measurement Technology Conference 688 Proceedings, Vol 1, 2005, pp. 257-260

689 Rivas J. J., Lobato R. D., Sarabia R., Cordobés F., Cabrera A., \& Gomez P., "Extradural 690 Hematoma: Analysis of Factors Influencing the Courses of 161 Patients," in Neurosurgery, Vol. 691 23, 1998, pp. 44-51

692 Roelens L., Van den Bulcke S., Joseph W., Vermeeren G., \& Martens L. "Path loss model for 693 wireless narrowband communication above flat phantom in Electronics Letters, IET, Vol. 42, 694 2006, pp. 10-11

695 Sanchez S., "Phantom studies of micro-impulse radar detection of cranial hematomas,", M.S 696 Thesis, Electrical and Electronic Engineering, California State University, Sacramento, 2013.

697 Shaver E. G., Duhaime A. C., Curtis M., Gennarelli L. M., \& Barrett R., "Experimental acute 698 subdural hematoma in infant piglets in Pediatric neurosurgery, Vol. 25, 1996, pp. 123-129

699 Teichmann D., Kuhn A., Leonhardt S., \& Walter M., "The main shirt: A textile-integrated 700 magnetic induction sensor array," in Sensors (Switzerland), Vol 14, 2014, pp. 1039-1056

701 Teichmann D., Foussier J., Jia J., Leonhardt S., \& Walter M., "Noncontact monitoring of 702 cardiorespiratory activity by electromagnetic coupling," in IEEE Trans. Biomed. Eng, Vol 60, 703 2013, pp. 2142-2152 
704 Van Beek A. H., Claassen J. A., Rikkert M. G. O., \& Jansen R. W., "Cerebral autoregulation: an 705 overview of current concepts and methodology with special focus on the elderly." In Journal of 706 Cerebral Blood Flow \& Metabolism, Vol 28, 2008, pp. 1071-1085.

707 Velander J., Redzwan S., Perez M. D., Asan N. B., Nowinski D., Lewén A., \& Augustine R., “A 708 Four-Layer Phantom for Testing In-Vitro Microwave-Based Sensing Approach in Intra-Cranial 709 Pressure Monitoring," in IEEE, International Microwave Biomedical Conference (IMBioC), 2018, 710 pp. $49-51$.

711 Zentgraf H., Deumling B., \& Franke W. W., "Isolation and characterization of nuclei from bird 712 erythrocytes in Elsevier, Experimental cell research, Vol. 56, 1969, pp. 333-337.

713 Zhao S., Li G., Gu, S., Ren J., Chen J., Xu L. \& Sun, J., "An Experimental Study of Relationship 714 Between Magnetic Induction Phase Shift and Brain Parenchyma Volume With Brain Edema in 715 Traumatic Brain Injury," in IEEE Access, vol. 7, 2019, pp. 20974-20983, doi: 716 10.1109/ACCESS.2019.2897609.

717 Zhou C., Eucker S. A., Durduran T., Yu G., R., Friess S. H., Ichord R. N., Margulies S. S., Yodh 718 A. G., "Diffuse optical monitoring of hemodynamic changes in piglet brain with closed head injury 719 in International Society for Optics and Photonics, Journal of biomedical optics, Vol. 14, 2009, p. 720034015. 
Figure 1

Scheme of the experimental network, $\mathrm{S}_{11}$ diagram of the coil, and visualization of the elements in the experiment.
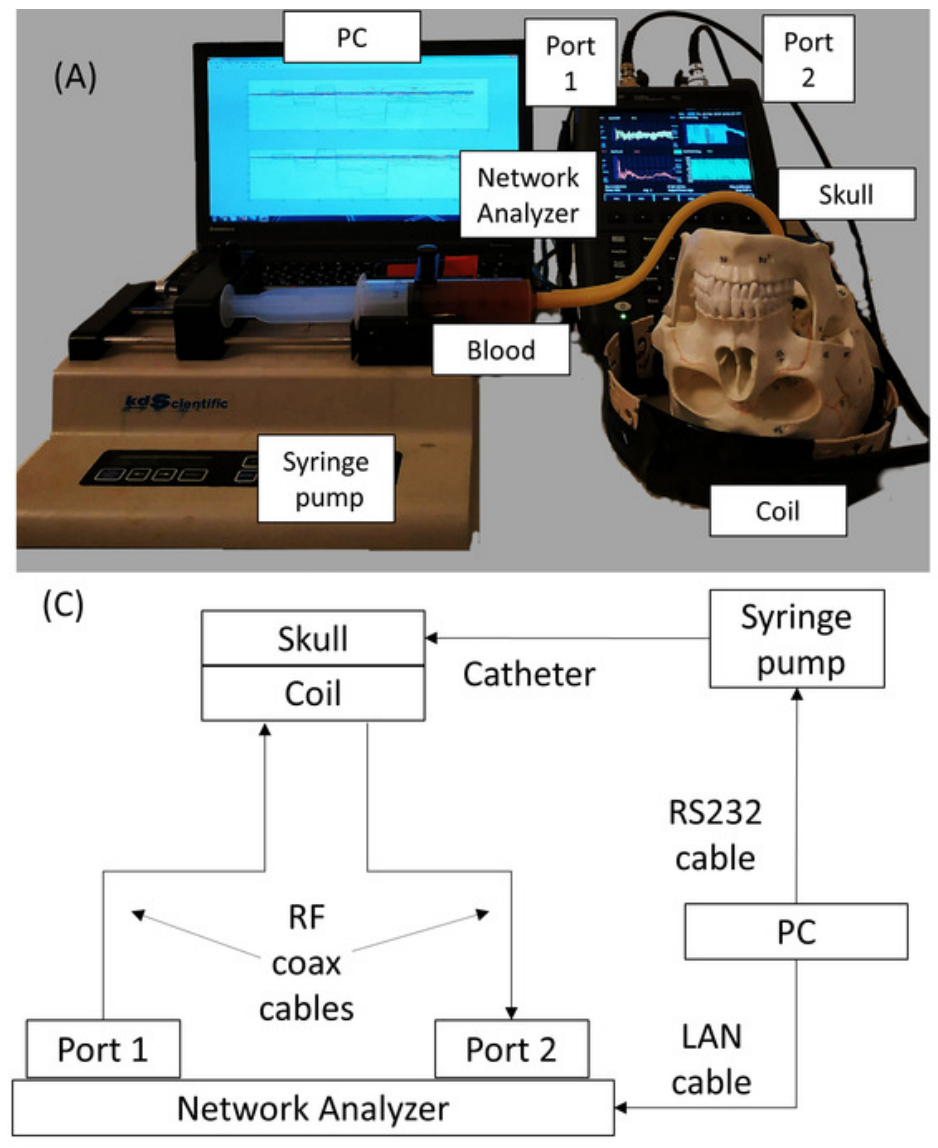
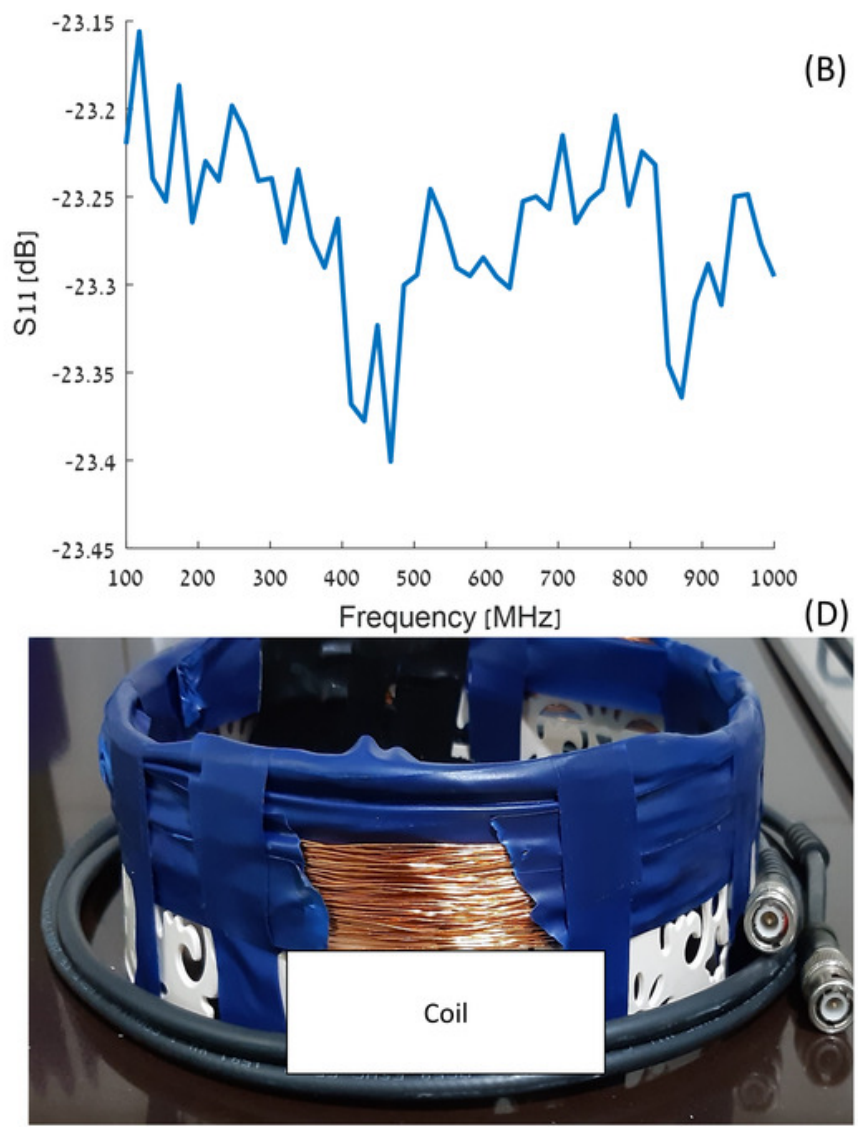
Figure 2

Steps in preparing the skull for an experiment $(a, b)$, and the blood injections sites $(c, d)$
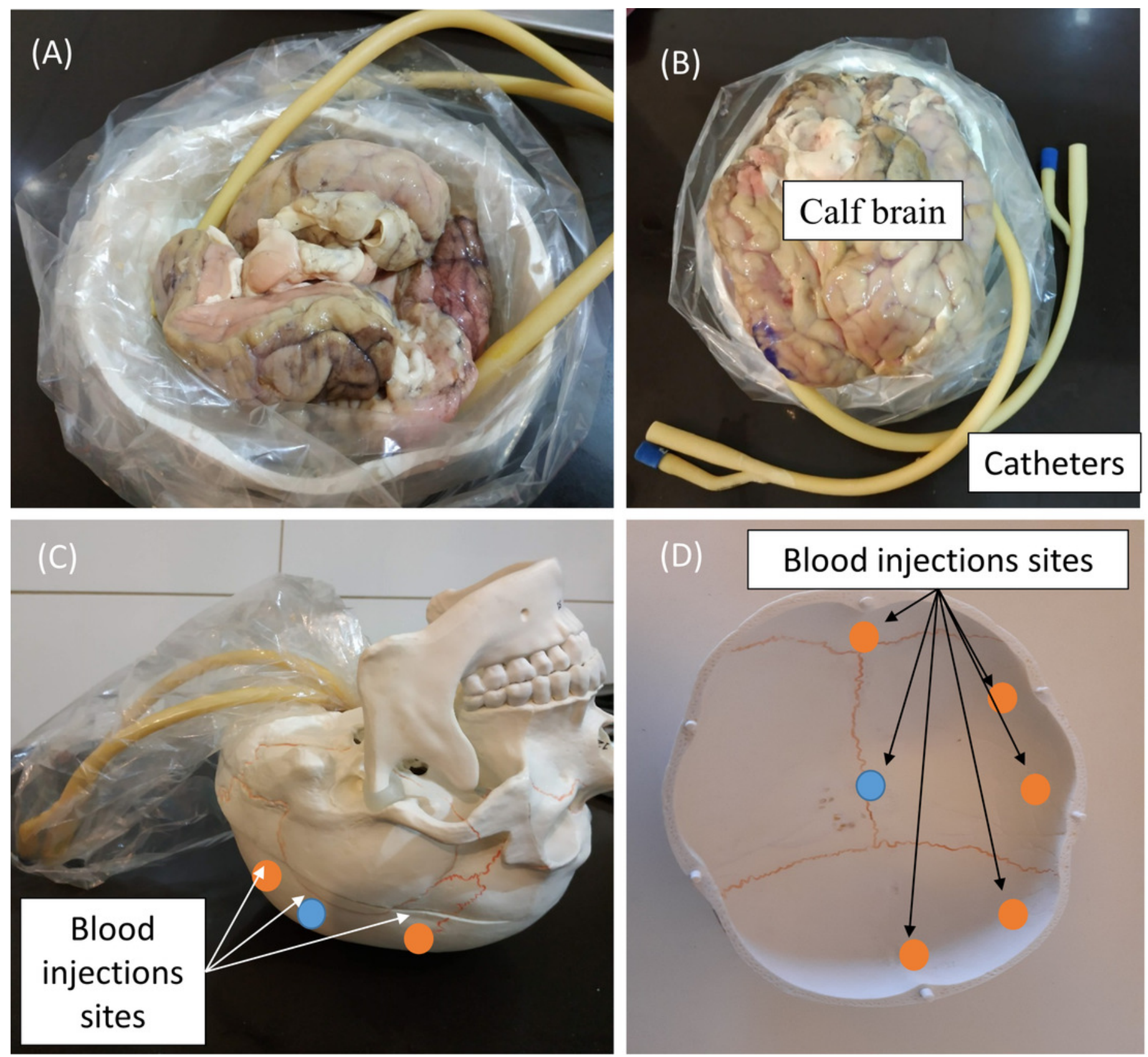

(D)

Blood injections sites

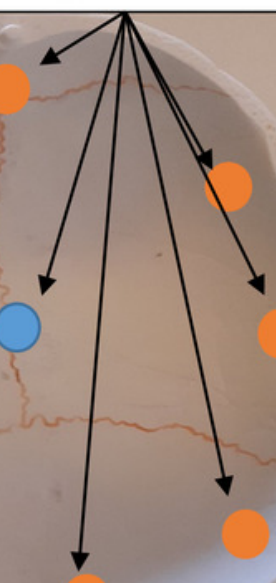


Figure 3

Examples of the changes in amplitude (left) and phase (right) in response to incremental increase in the volume of injected blood at the center of the skull, as a function of three different arbitrary chosen frequencies.

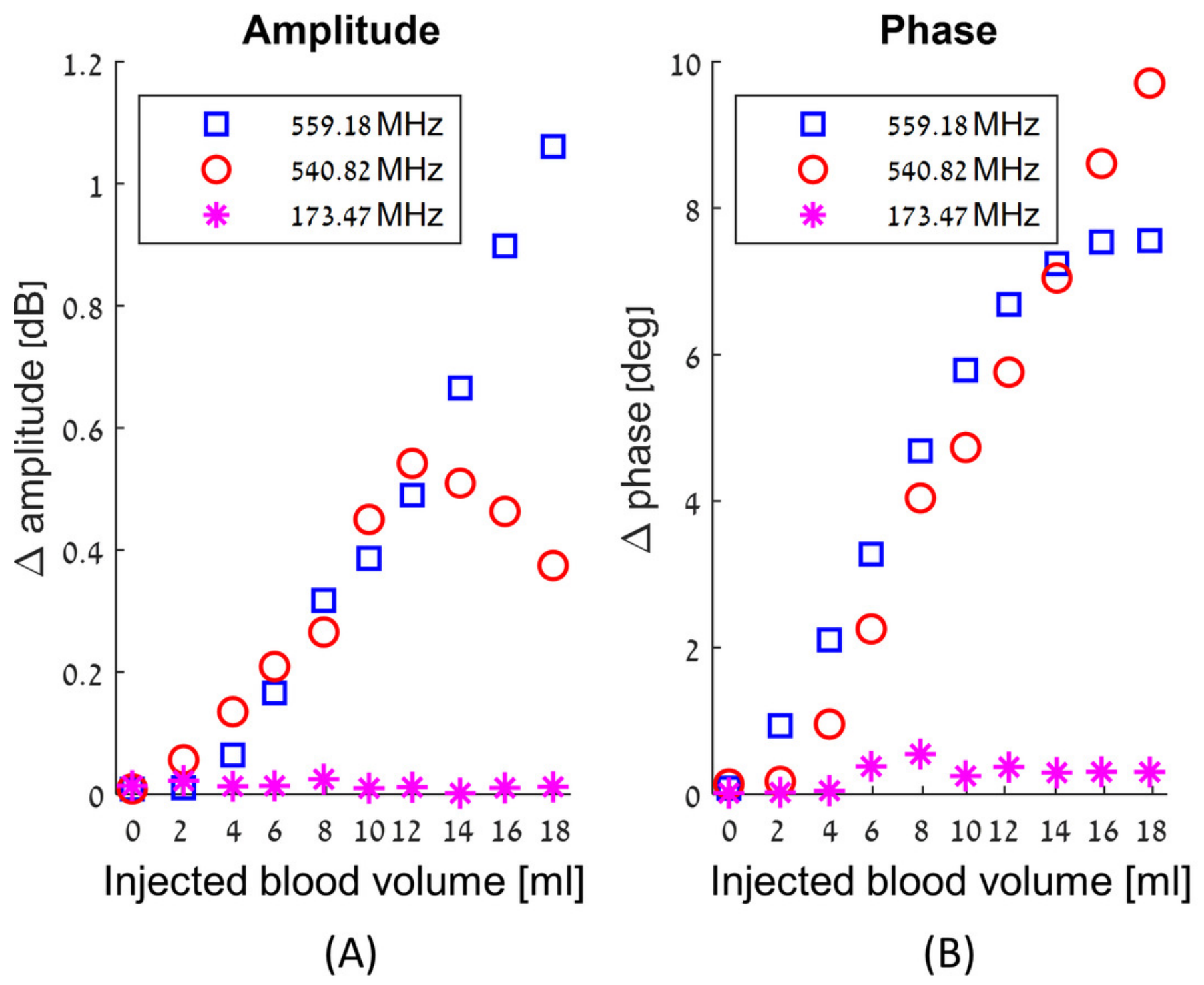


Figure 4

Histograms of $R(F)(a, c)$ and $\log _{10}(R(F))(b, d)$ for the amplitude $(a, b)$ and phase $(c, d)$.

A normal distribution plot is shown for comparison (red line). The normal distribution of (d) had fitted on the data without the extreme values ( (the light blue bars).
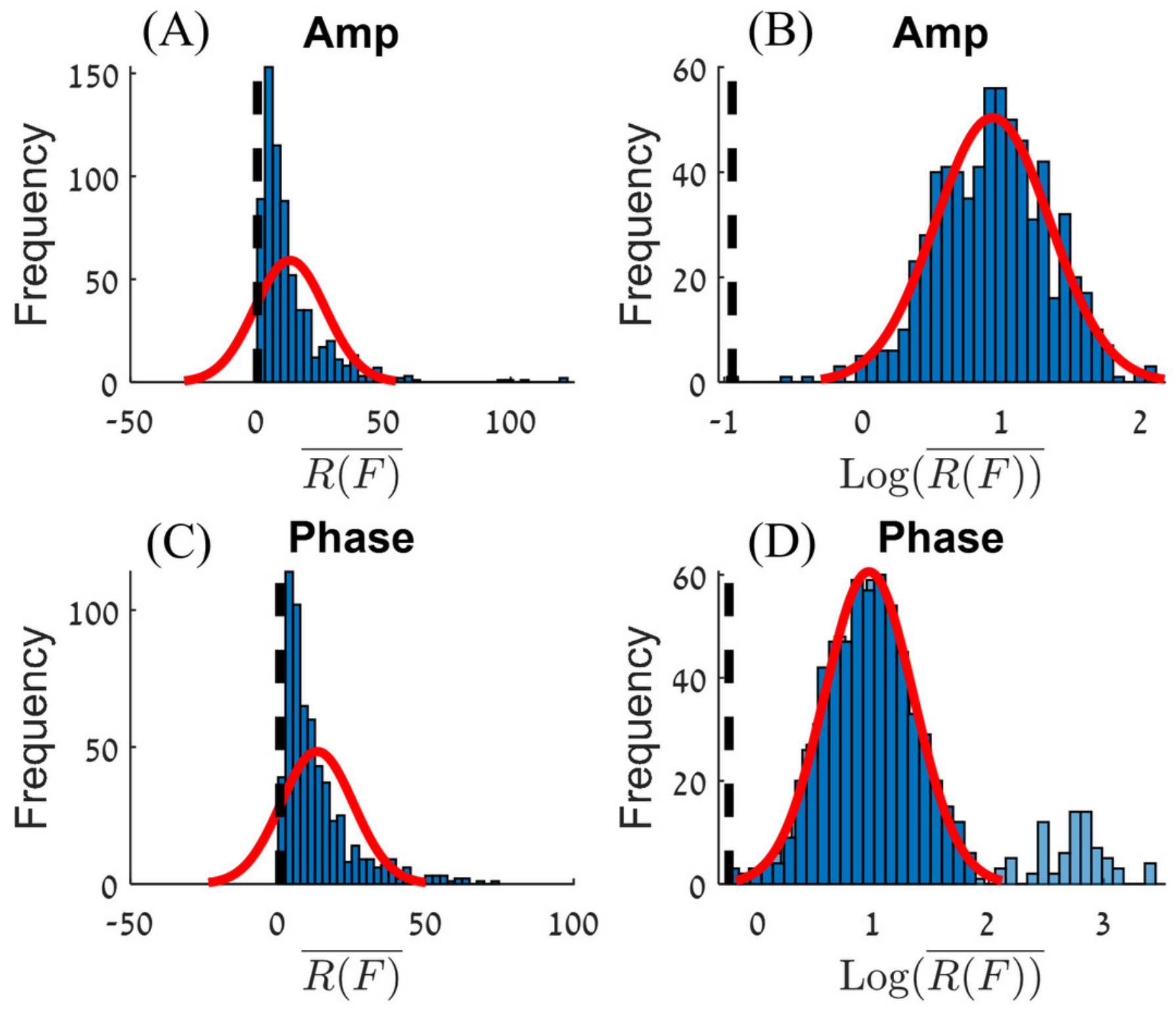
Figure 5

$\log _{10}(R(F)$ ) values for amplitude (a) and phase (b) at a boxplot format (without the outliers).

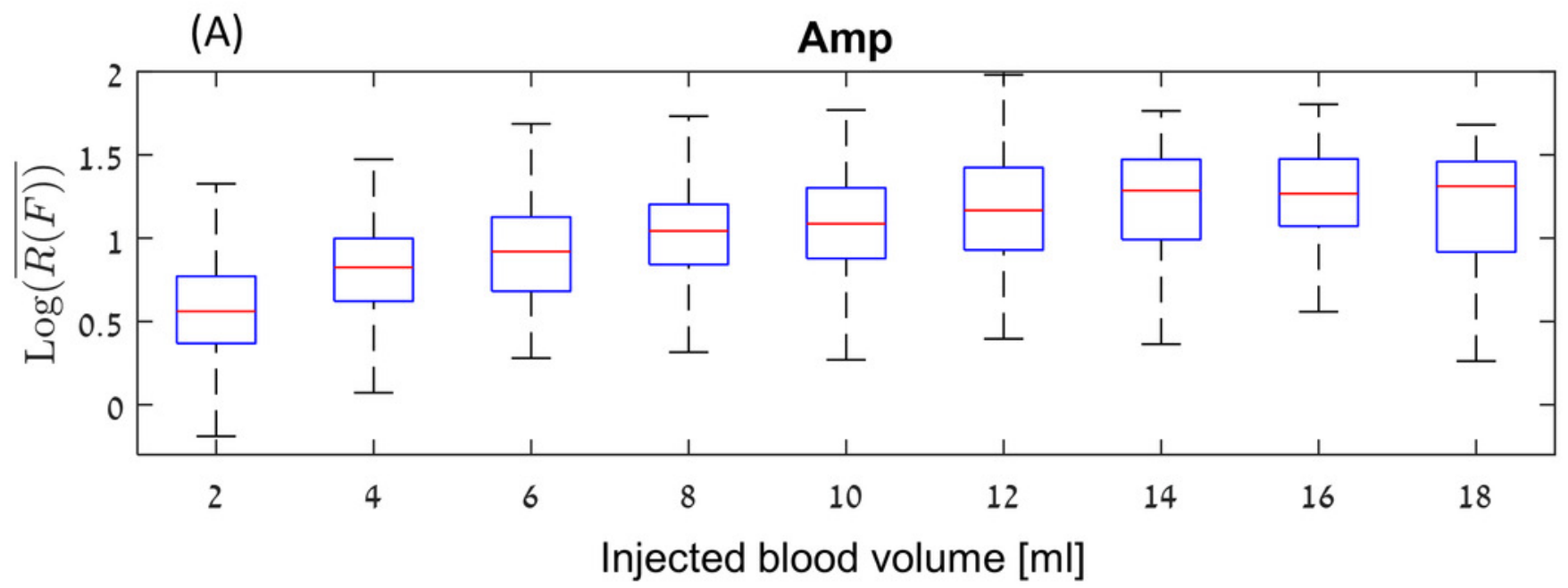

(B)

Phase

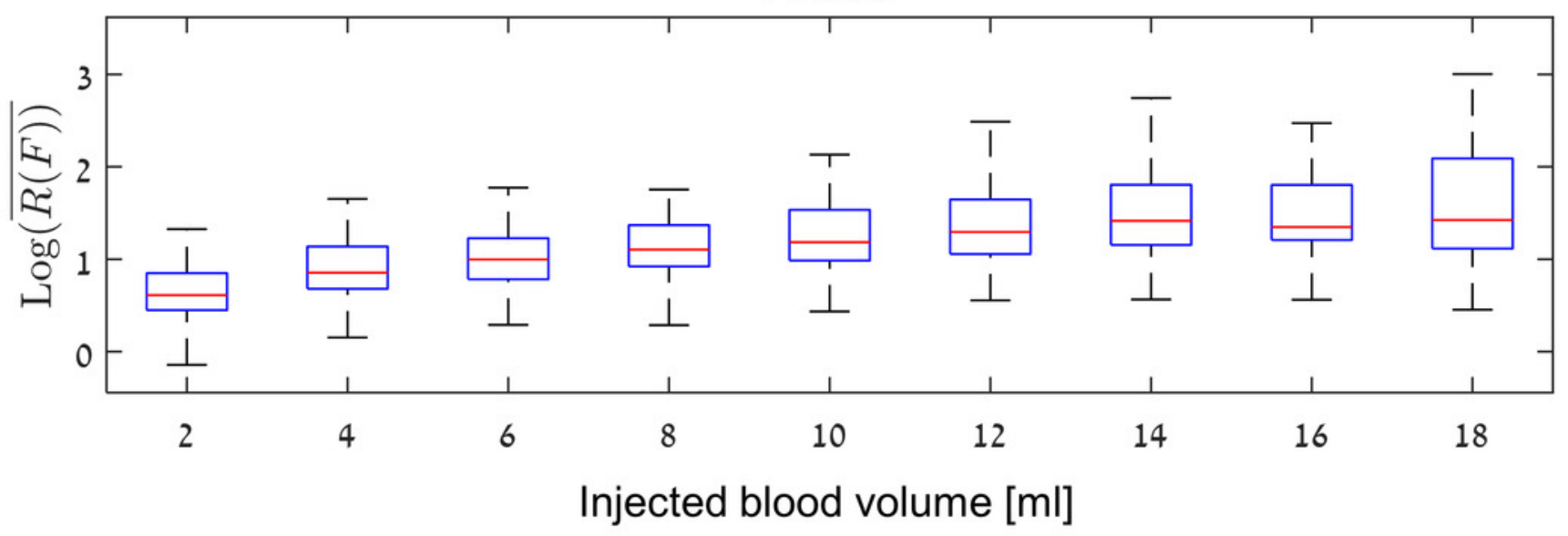




\section{Figure 6}

$\log _{10}(R(F))$ for the amplitude versus $\log _{10}(R(F))$ for the phase

The experiments in which the blood was injected in/out the balloon marked with green markers, experiments in which the blood was injected into a balloon marked with blue markers and experiments in which the blood was injected directly into the brain marked with red markers.

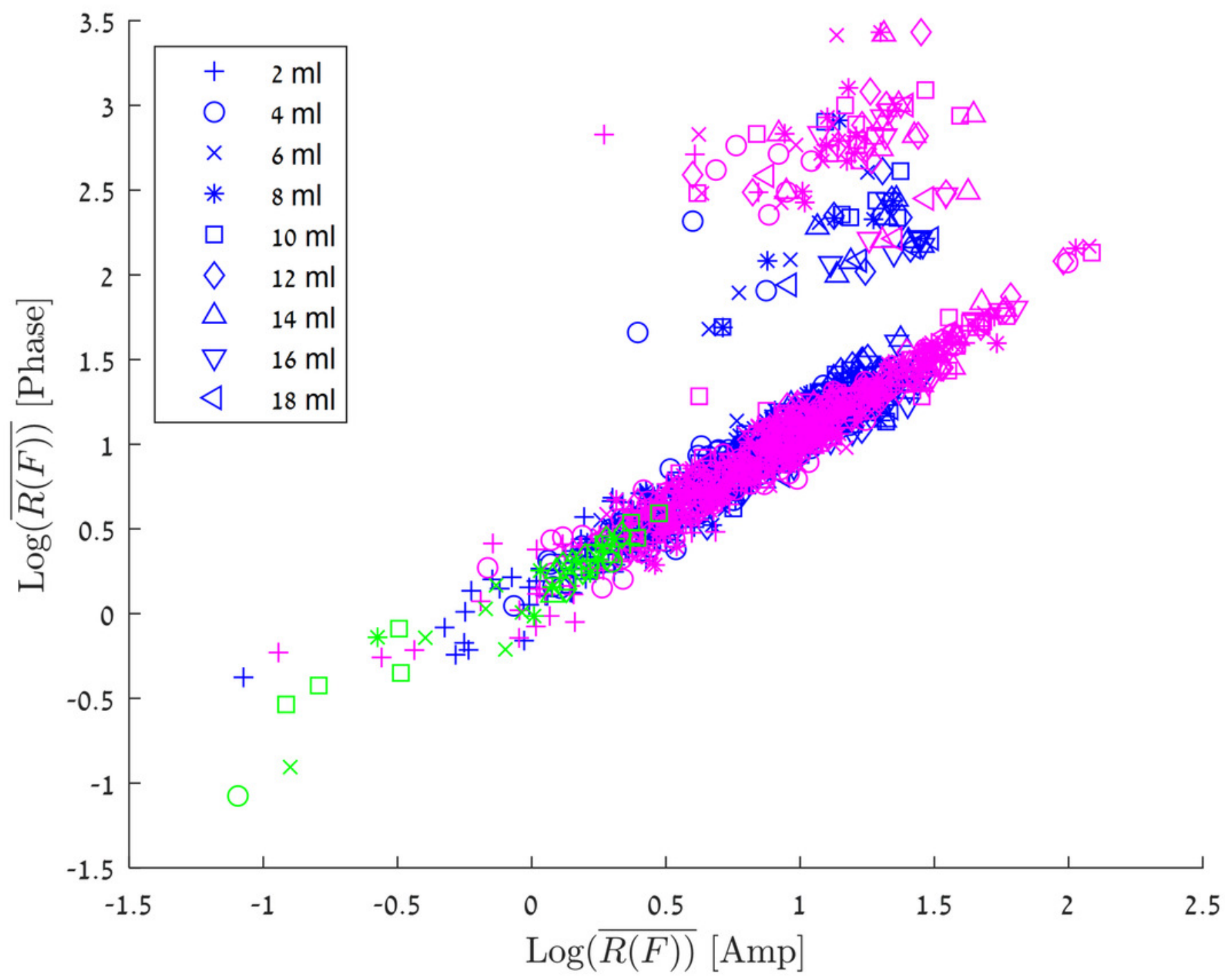


Figure 7

Linear regression model for the injection volume based on the amp/phase values.

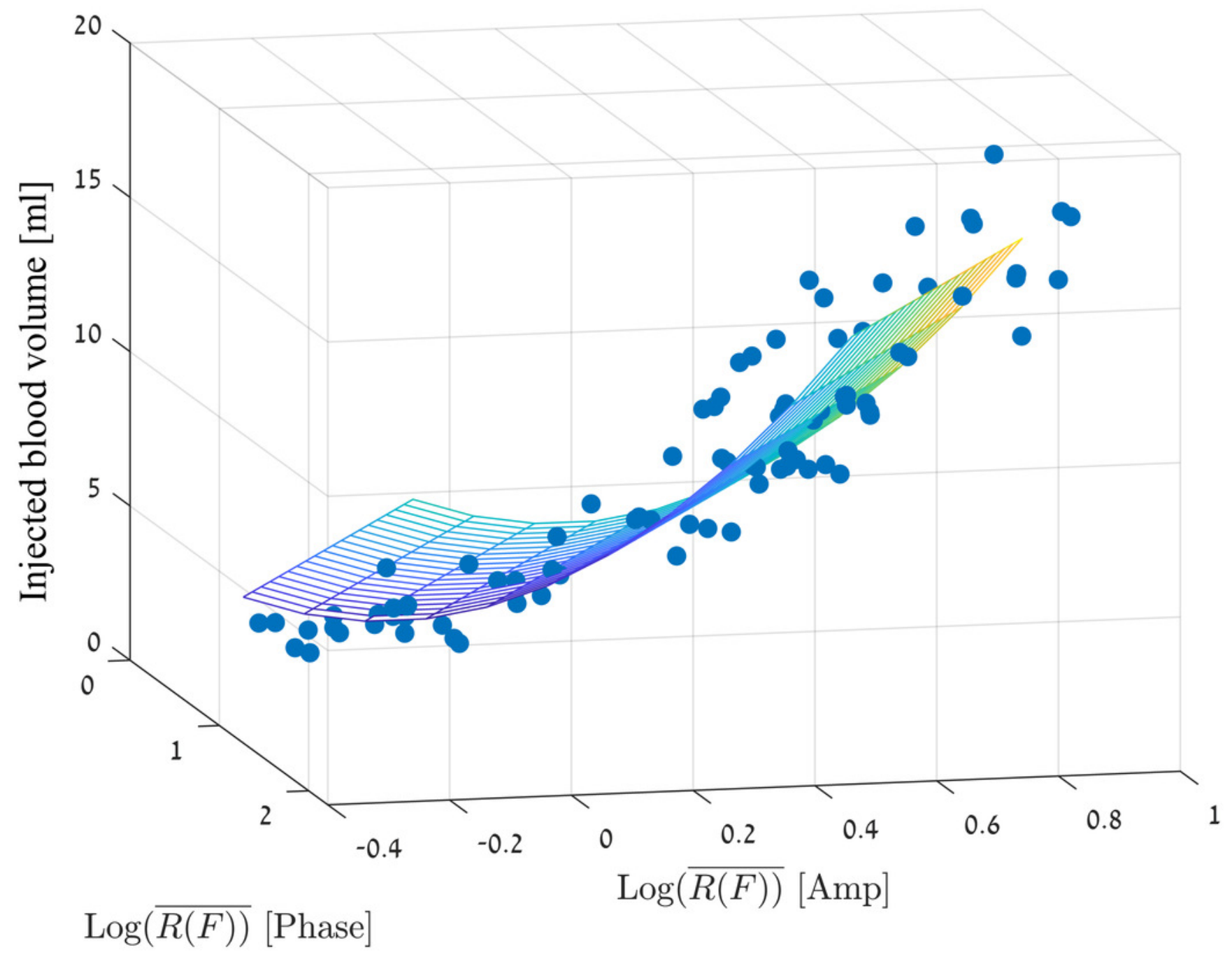


Figure 8

Estimated volume versus injected volume. The results are given as mean $\pm \mathrm{SD}$

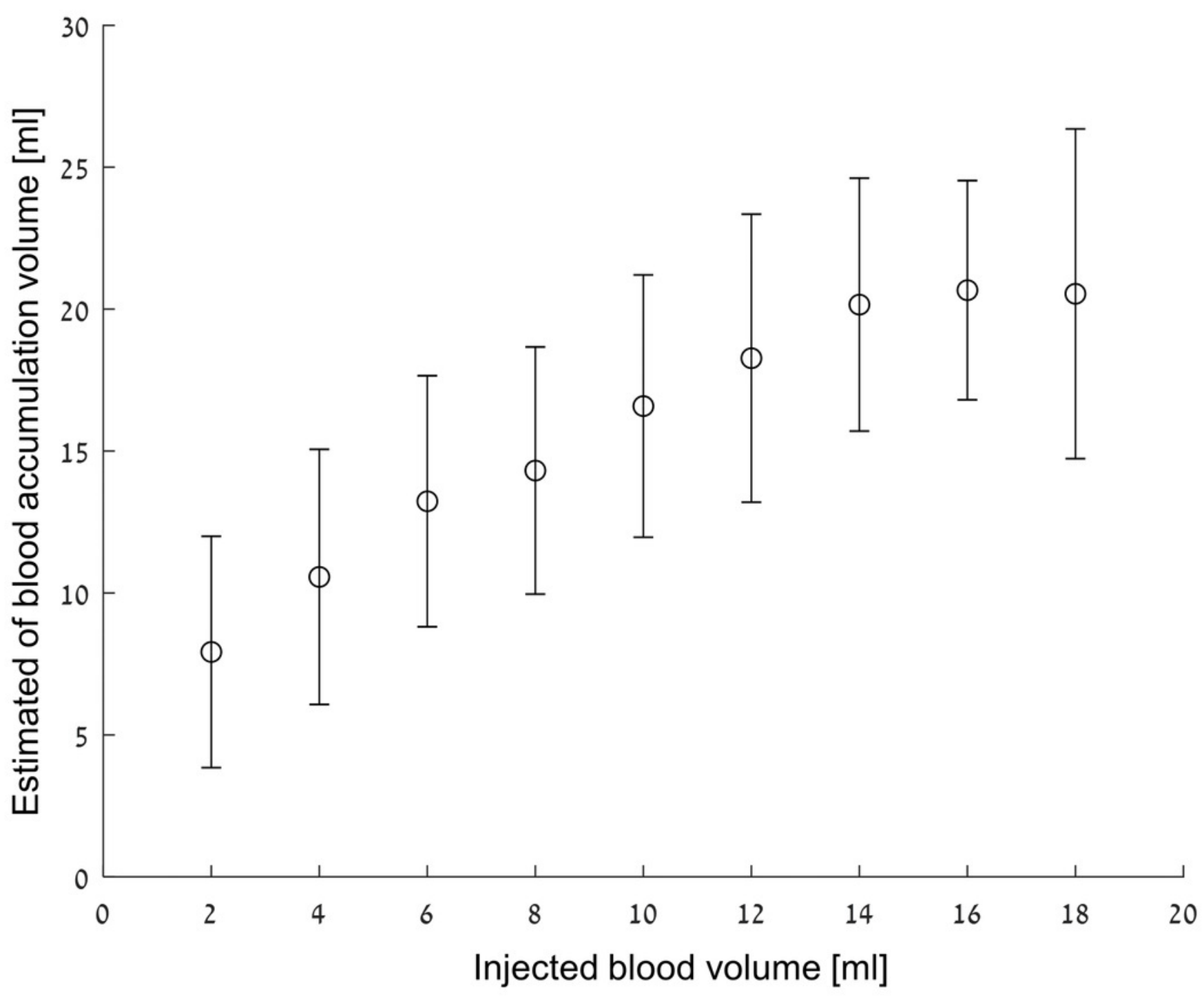




\section{Table $\mathbf{1}$ (on next page)}

Dielectric parameters

The human brain, skin and the blood dielectric properties derived from (Hasgall et al., 2018). Chicken blood properties derived from (Kratzenberg et al., 2003). Bovine brain properties were derived from (Gabriel et al., 1996). PVC properties were derived from (Chung, K.T. et al., 1982) and saline properties derived from (Alanen, 1999). 


\begin{tabular}{|c|c|c|c|c|c|c|c|c|c|c|c|c|c|c|c|c|}
\hline \multirow[b]{2}{*}{ Frequency } & \multicolumn{2}{|c|}{ Human brain } & \multicolumn{2}{|c|}{ Human skin } & \multicolumn{2}{|c|}{ Bovine brain } & \multicolumn{2}{|c|}{ Human blood } & \multicolumn{2}{|c|}{ Chicken blood } & \multicolumn{2}{|c|}{ Saline } & \multicolumn{2}{|c|}{ Human Skull } & \multicolumn{2}{|c|}{$\mathrm{PVC}$} \\
\hline & $\varepsilon_{r}$ & $\sigma$ & $\varepsilon_{r}$ & $\sigma$ & $\varepsilon_{r}$ & $\sigma$ & $\varepsilon_{r}$ & $\sigma$ & $\varepsilon_{r}$ & $\sigma$ & $\varepsilon_{r}$ & $\sigma$ & $\varepsilon_{r}$ & $\sigma$ & $\varepsilon_{r}$ & $\sigma$ \\
\hline $100 \mathrm{MHz}$ & 89.76 & 0.79 & 72.9 & 0.49 & 94.2 & 0.80 & 76.8 & 1.23 & 80.2 & 0.92 & 120 & 1.48 & 15.28 & 0.06 & 8.5 & $<10^{-3}$ \\
\hline $500 \mathrm{MHz}$ & 53.82 & 1.08 & 49.9 & 0.72 & 47.0 & 1.10 & 63.3 & 1.38 & 65.8 & 1.41 & 61 & 1.51 & 12.95 & 0.10 & 9.1 & $<10^{-3}$ \\
\hline $1000 \mathrm{MHz}$ & 48.85 & 1.30 & 40.9 & 0.90 & 41.6 & 1.30 & 61.1 & 1.58 & 63.9 & 1.60 & 55 & 1.56 & 12.36 & 0.15 & 9.3 & $<10^{-3}$ \\
\hline
\end{tabular}

